\title{
BcMF27, a Pectin Methylesterase Gene, Regulates Pollen Development And Pollen Tube Growth in Brassica Campestris
}

\section{Xiaoyan Yue}

Hainan Institute of Zhejiang University

Jiashu Cao ( $\sim$ jshcao@zju.edu.cn )

Hainan Institute of Zhejiang University https://orcid.org/0000-0002-8637-987X

\section{Research Article}

Keywords: Brassica campestris, PMEs, pollen, pollen tube, intine

Posted Date: November 1st, 2021

DOI: https://doi.org/10.21203/rs.3.rs-725136/v2

License: (c) (i) This work is licensed under a Creative Commons Attribution 4.0 International License.

Read Full License 


\section{Abstract}

Functional pollen grains are an essential ingredient of successful reproduction in flowering plants and are protected by outer walls. Pectin methylesterases (PMEs) modify pectin, a structural component of pollen intine. However, there are few studies on PMEs. Artificial microRNA (amiRNA) and overexpression technology was performed to investigate the function of pollen-specific PME gene, BcMF27, in pollen development. Knockdown of BCMF27led to pollen wall collapse, $20 \%$ of which unknown material adhered to. Wall-collapsed pollen had abnormally thick intine outside of the germinal furrows. A portion of the cytoplasm was degraded in the remaining pollen with unknown material on the wall, in addition to a thick intine. Overexpression of BcMF27 resulted in $66.67 \%$ pollen wall disruption, causing an abnormally thick intine. In addition, functional interruption of BcMF27 gave rise to pollen tubes twisted in vitro. Taken together, BCMF27 contributes to the intine morphogenesis during pollen development and stabilizes pollen tube elongation. This research can promote knowledge of PMEs function and the molecular mechanism in pollen wall construction.

\section{Key Message}

\section{Unbalanced expression of BcMF27 induced pollen morphology defects and pollen tubes twist. Therefore, BcMF27 contributes to pollen construction and maintains pollen tube stability.}

\section{Introduction}

Pollen wall is the outer structure of pollen grain, protecting the male gametophyte from physical and biological stress. The pollen wall plays an important role in pollen development, the key procedure of the angiosperm life cycle (Zhang et al. 2016). The elaborately organized pollen wall consists of the exine and intine. The major component of the exine is sporopollenin. The intine comprises pectin, structural proteins, and microfibrillar cellulose (Huang et al. 2009). Pectin is a structurally covalently linked heteropolysaccharide (Mohnen 2008). PMEs (EC 3.1.1.11) catalyze demethylation of HG at the C-6 to release the free negatively charged carboxyl group and reduce the degree of methylesterification (DM) (Pelloux et al. 2007). De-esterified pectin molecules link to each other by calcium ions to form an "egg-box" supermolecular gel (Yoneda et al. 2010). The pectic gel is then integrated into the wall to strengthen wall rigidity (Roger et al. 2001). Therefore, the demethylation of pectin by PMEs affects pollen development by participating in wall construction.

PMEs exist ubiquitously in the whole plant life cycle (Pelloux et al. 2007) and have important biological significance in plant growth and development, including pollen development, cell elongation, stem morphogenesis, cell adhesion and separation, seed coat mucilage extrusion, abiotic stress, and biological stress (Micheli 2001; Hongo et al. 2012; Sénéchal et al. 2014; Levesque-Tremblay et al. 2015; Turbant et al. 2016; Yue et al. 2018a). PMEs belong to a large gene family (Markovič and Janeček 2004), and some PME genes are expressed specifically in pollen grains, for example, NtPPME1 from Nicotiana tabacum (Bosch and Hepler 2006; Wang et al. 2013) and AtPPME1 and VGD1 from Arabidopsis thaliana (Jiang 
2005; Tian et al. 2006; Chen and Ye 2007), which have been confirmed to affect pollen tube growth. Moreover, Francis et al. (2006) observed that QRT1 was involved in the separation of tetrad. AtPME48 regulated pollen germination (Leroux et al. 2015). BcMF23a contributed to pollen development and pollen tube growth (Yue et al. 2018a). BCPME37c influenced pollen intine formation (Xiong et al. 2019). However, studies on the biological function of PMEs, particularly in pollen development, still remain limited.

Previously, BcMF27 from B. campestris was identified as a putative pectin methylesterase gene. BcMF27 was expressed significantly in mature pollen and pollinated pistils (Yue et al. 2018b). In this study, artificial microRNA (amiRNA) and overexpression technology was used to further determine the biological function of BCMF27. Knockdown and overexpression of BCMF27 led to intine formation outside of the germinal furrows, pollen morphology abortion, and pollen tube twisting. According to these results, it is suggested that BCMF27 is necessary for pollen development and pollen tube growth by participating in pollen intine and pollen tube wall, respectively.

\section{Materials And Methods}

\section{Construction of vectors and plant transformation}

The amiRNA sequence was designed according to the procedure in the Web MicroRNA Designer (WMD3Web MicroRNA Designer; 5'-TAAGCAACATACACTGCGCGA-3'), and miR164a was used as the gene backbone. The incorporated sequence was integrated into the binary vector pCAMBIA1301 between Xbal and Hindll with a constitutive CaMV35S promoter. The cDNA sequence of BcMF27(Yue et al. 2018b) was cloned and inserted into the same vector between the restriction sites. Then, amiRNA, overexpression, and empty vectors were induced in B. campestris ssp. chinensis var. parachinensis by Agrobacterium tumefaciens to obtain transgenic lines according to Yu et al. (2004). Transgenic and control plants were cultivated in an illumination incubator at $22^{\circ} \mathrm{C}$ with a photoperiod of $16 \mathrm{~h}$ light $/ 8 \mathrm{~h}$ dark.

\section{Detection of positive transgenic plants}

To identify the transformed lines, PCR was performed with genomic DNA extracted from fresh young leaves using the primers 5'-CCAGGCTTTACACTTTATGC-3' and 5'-GCGATTAAGTTGGGTAACGC-3'. Total RNA of the whole inflorescence from positive plants was extracted by the Trizolß reagent (Invitrogen, Carlsbad, CA, USA) and then used to synthesize the first-strand CDNA with the PrimerScript RT reagent kit (TaKaRa, Japan). Real-time RT-PCR was performed with the SYBR Premix Ex Taq Kit (TaKaRa, Japan) using a Bio-Rad CFX96 Real-time PCR Detection System (Bio-Rad, USA). UBC10 was used as a control. The primer sets were as follows: BcMF27F 5'-ATGGCGTTTCAGGATTTCGAC AA-3' and BcMF27R 5'TCACGCATCATAAAGACCAAGC-3'; and UBC10F 5'-GGGTCCT ACAGACAGTCCTTAC-3' and UBC10R 5'ATGGAACACCTTCGTCCTAAA-3'. Three biological repeats were performed. The $2^{-\triangle \triangle C t}$ method was used to analyze the relative expression levels of BcMF27 (Livak and Schmittgen 2001). 


\section{Pollen microscopy observation}

Scanning electron microscopy (SEM) of mature pollen and transmission electron microscopy (TEM) of pollen during pollen development were performed according to Lin et al. (2014). Semi-thin anther sections $(2 \mu \mathrm{m})$ from anthers collected during pollen development were stained by dimethyl blue and photographed with a fluorescent microscope (Leica, Germany). The pollen abortion percentage was analyzed.

\section{Pollen germination in vitro}

Pollen germination in vitro was performed according to Lin et al. (2014). Pollen tubes were observed and photographed with a fluorescent microscope. The rate of pollen germination and percentage of abnormal pollen tubes were calculated. Three biological repeats were performed.

\section{Results}

\section{Up- and downregulation of BcMF27 resulted in pollen abnormality}

To determine the function in pollen development, amiRNA and overexpression vectors of BcMF27 were constructed (Fig. 1a, b) and induced in B. campestris ssp. chinensis var. parachinensis. Positive transgenic lines were confirmed by PCR analysis and named BcMF27-amiR and BCMF27OE (Fig. 1c). The $B C M F 27$ transcripts from the inflorescence of BCMF27-amiR and $B C M F 27^{O E}$ plants were detected by realtime RT-PCR (Fig. 1d). The results showed that the expression of BCMF27 decreased in the inflorescence of BcMF27-amiR1-3 plants and increased in the inflorescence of $B C M F 27^{O E}-1-3$ plants although variation of the BcMF27 transcript was not remarkable.

No difference was observed in vegetative growth and flower organs development among BcMF27-amiR and $B C M F 27^{O E}$ and control transgenic plants (Suppl. Fig. S1). However, SEM indicated that the pollen of BcMF27-amiR and BCMF27OE plants was defective (Fig. 2). Of pollen from BcMF27-amiR plants, $93.56 \%$ possessed collapsed walls (Fig. $2 b, e, f, h$ ). The surface of $29 \%$ of abnormal pollen walls locally accumulated unknown material (Fig. 2e). Of $B C M F 27^{O E}$ pollen, $66.67 \%$ had collapsed walls (Fig. 2c, g, i). Pollen from control plants had an ellipsoid shape and normal reticular structure, and the deformity rate was only $13.75 \%$ (Fig. 2a, d, h, i).

To clarify anther and pollen development in BcMF27-amiR and $B C M F 27^{O E}$ transgenic plants, semi-thin sections of BCMF27-amiR and $B C M F 27^{O E}$ pollen were collected (Fig. 3). Tapetum development in BcMF27-amiR (Fig. $3 f-j$ ) and BCMF2 $2{ }^{O E}$ (Fig. 3k-o) plants was identical to that of control plants (Fig. $3 a-e)$. BcMF27-amiR (Fig. $3 f-i)$ and $B C M F 27^{O E}$ (Fig. $3 k-n$ ) pollen developed normally from the pollen mother cell stage to the binucleate stage. However, BcMF27-amiR pollen (Fig. 3j) exhibited two kinds of malformed shapes and $B C M F 27^{O E}$ pollen (Fig. 30) presented an irregular shape compared with control pollen at the trinucleate stage (Fig. $3 e$ ). 
To further detail BcMF27-amiR and BCMF27OE pollen development, TEM was performed (Fig. 4). BcMF27amiR pollen development normally proceeded to the uninucleate stage (Fig. $4 f-h, r$ ) in accordance with the control (Fig. 4a-c, q). At the binuclear stage, the intine thickened normally at germinal furrow regions in control pollen (Fig. 4d, t), but $95.45 \%$ of BcMF27-amiR pollen intines formed abnormally outside germinal furrows (Fig. 4i, u). Furthermore, $36.36 \%$ of BcMF27-amiR pollen with an additional germinal aperture displayed partial cytoplasm degradation and unknown material on the surface of pollen (Fig. $4 \mathrm{j}$, $x$ ) and the remaining defective pollen still included four germinal furrows (Fig. $4 p$, z), while the control pollen contained a normal organized wall with three germinal furrows and a dense cytoplasm (Fig. 4e, w) at the trinucleate stage. $B C M F 27^{O E}$ pollen showed normal development from the pollen mother cell stage to the binuclear pollen stage (Fig. $4 \mathrm{k}-\mathrm{n}, \mathrm{s}, \mathrm{v}$ ), whereas $52 \%$ of pollen exhibited aberrant intine deposition outside the germinal furrow regions at the mature pollen stage (Fig. 40, y).

\section{Functional disruption of BcMF27 caused morphologically unstable pollen tubes}

Alexander staining was performed for pollen viability analyses (Suppl. Fig. S2). It was observed that, similar to control pollen (Suppl. Fig. S2a), BcMF27-amiR (Suppl. Fig. S2b) and BcMF270E (Suppl. Fig. S2c) pollen grains were purplish red, which indicated normal BcMF27-amiR and BcMF27 ${ }^{\mathrm{OE}}$ pollen viability.

In BcMF27-amiR and $B C M F 27^{O E}$ transgenic plants, pollen germination in vitro was checked to determine the effect of BCMF27 expression imbalance on pollen germination and pollen tube growth (Fig. 5). The average germination rates of $B C M F 27-a m i R$ and $B C M F 27^{O E}$ pollen were $74.95 \%$ and $71.86 \%$, respectively. The pollen germination of BCMF27-amiR and $B C M F 27^{O E}$ was hardly affected compared with control pollen (80.68\%; Fig. 5d). However, BcMF27-amiR (Fig. 5b) and BCMF27OE (Fig. 5c) had twisted tubes compared with the positive control (Fig. 5a). The percentage of twisted pollen tubes in BcMF27-amiR1, BcMF27-amiR2, and BcMF27-amiR3 (91.67\%, 85.22\%, and 94.58\%, respectively) were significantly increased (Fig. 5d). Similarly, the percentage of twisted pollen tubes was significantly increased in $B C M F 27^{O E_{-}}$, BCMF2 $2 \mathrm{OE}_{-2}$, and $B C M F 27^{O E_{-}} 3(50 \%, 78.33 \%$, and $41.11 \%$, respectively; Fig. 5 d). However, pollen from BCMF27-amiR (Fig. 6b) and $B C M F 27^{O E}$ (Fig. 6c) on control pistils germinated normally in vivo compared to that from control (Fig. $6 \mathrm{a}$ ) at $4 \mathrm{~h}$ after pollination. The average seed number per silique from BcMF27-amiR, BCMF27OE, and control plants was not significantly different (data not shown).

\section{Discussion}

The elaborately decorated pollen wall protects pollen from stress and consists of the exine and intine. Pectin is a structural component of the intine and is involved in the modification of wall characteristics. PMEs hydrolyze pectin and demethylate pectin molecules linked to each other by calcium ion bonds; they are then integrated into the intine to strengthen wall rigidity. Therefore, PMEs have an essential function in pollen wall construction and pollen development. 
Yue et al. (2018a) observed that knockdown of BcMF23a caused shape deformities and defective intine construction in pollen. Therefore, it was inferred that BcMF23a influenced pollen development via intine formation. BCMF27, the characteristic pollen-specific pectin methylesterase, was remarkably expressed in mature pollen (Yue et al. 2018b). BcMF27-amiR and BCMF2 $7^{O E}$ mature pollen walls collapsed and the intine thickened abnormally outside of the germinal apertures induced by variation of the BcMF27 transcript, which was similar to the phenotype of bcmf23a pollen. In addition, in 36.36\% of BcMF27-amiR pollen, the cytoplasm partially disappeared, which also occurred in bcmf23a pollen. Thus, it was estimated that the imbalance of $B C M F 27$ expression led to abnormal intine formation, which further affected pollen morphology construction.

The pollen tube is an extension of the pollen intine and grows rapidly in the pistil (Geitmann and Steer 2006). Pollen tube walls are mainly composed of pectin (Chen and Ye 2007), which control the inflexibility of pollen tubes. The remodeling of the pollen tube wall involves many enzymes, such as glycoside hydrolases (GHs), xyloglucan endo-transglucosylase hydrolase (XTHs), and PMEs (Yokoyama and Nishitani 2001; Tung et al. 2005; Mollet et al. 2013). PMEs regulate the DM of pectin to adjust the mechanical properties of the pollen tube, such as stiffness and elasticity (Staunton et al. 2011; Chebli et al. 2012; Zhang et al. 2018).

Many pollen-specific PME genes have demonstrated an important function in pollen germination and pollen tube growth (Kim et al. 2020). AtPME48 belongs to the pollen-specific PME gene (Leroux et al. 2015). Functional interruption of AtPME48 resulted in the appearance of two pollen tubes in vitro, delayed growth of pollen tubes in the pistil, and increased the DM of pme48 pollen grains. It was concluded that AtPME48 affected pollen germination by influencing the formation of the pollen intine. VGD1 was also expressed in pollen and the pollen tube (Jiang 2005). VGD1 knockout caused the pollen tube to burst in vitro, and pollen tube growth in vivo was postponed, causing the reduction of PME activity in the pollen tube, although the pollen grain displayed normal morphology. ZmGa1P and ZmPME10-1, two pollenspecific PMEs, assembled to modulate pectin esterification in pollen tube wall, which affected pollen tube growth (Zhang et al. 2018). Similarly, BCMF27 was specifically expressed in pollen and pollen tubes, and down- and upregulation of BCMF27led the tube to be significantly twisted. In addition, pollen fertility and germination in vitro/vivo were not affected by pollen defects incuced by the regulation of BcMF27. Therefore, according to these results, disruption of BCMF27 function directly reduced PME activity in the pollen tube, causing depressed rigidity in the tube wall, which destabilized pollen tube growth. The functional interruption of BCMF27 and BCMF23a led to intine construction abnormality, pollen morphology deformity, and pollen tube growth instability (Yue et al. 2018a). However, the transcript of BCMF27 appeared remarkably in pollen tubes, as well as mature pollen, while BcMF23a was expressed in pollen but not in the pollen tube (Lin et al. 2017; Yue et al. 2018a). Consequently, BcMF27not only was involved in intine formation to influence pollen development but also directly contributed to pollen tube growth by modifying pollen tube walls.

\section{Declarations}


Author contribution statement $\mathrm{JC}$ and $\mathrm{YY}$ conceived and designed the research. YY conducted the experiments, analyzed the data, and wrote the manuscript. JC revised the manuscript. All authors read and approved the final manuscript.

Funding This work was supported by the National Natural Science Foundation of China [No. 31772311 and No. 31471877]

Conflict of interest Not applicable.

\section{References}

1. Bosch M, Hepler PK (2006) Silencing of the tobacco pollen pectin methylesterase NtPPME1 results in retarded in vivo pollen tube growth. Planta 223:736-745. doi:10.1007/s00425-005-0131-x

2. Chebli Y, Kaneda M, Zerzour R, Geitmann A (2012) The Cell Wall of the Arabidopsis Pollen TubeSpatial Distribution, Recycling, and Network Formation of Polysaccharides. Plant Physiol 160:19401955. doi:10.1104/pp.112.199729

3. Chen LQ, Ye D (2007) Roles of pectin Methylesterases in pollen-tube growth. J Integr Plant Biol 49:94-98. doi:10.1111/j.1744-7909.2006.00418.x

4. Francis KE, Lam SY, Copenhaver GP (2006) Separation of Arabidopsis pollen tetrads is regulated by QUARTET1, a pectin methylesterase gene. Plant Physiol 142:1004-1013.

doi:10.1104/pp.106.085274

5. Geitmann A, Steer M (2006) The architecture and properties of the pollen tube cell wall. Plant Cell Monogr 3:177-200. doi:10.1007/7089_049

6. Hongo S, Sato K, Yokoyama R, Nishitani K (2012) Demethylesterification of the Primary Wall by PECTIN METHYLESTERASE35 Provides Mechanical Support to the Arabidopsis Stem. Plant Cell 24:2624-2634. doi:10.1105/tpc.112.099325

7. Huang L, Cao J, Zhang A et al (2009) The polygalacturonase gene BcMF2 from Brassica campestris is associated with intine development. J Exp Bot 60:301-313. doi:10.1093/jxb/ern295

8. Jiang L, Yang S-L, Xie L-F et al (2005) VANGUARD1 Encodes a Pectin Methylesterase That Enhances Pollen Tube Growth in the Arabidopsis Style and Transmitting Tract. Plant Cell Online 17:584-596. doi:10.1105/tpc.104.027631

9. Kim YJ, Jeong HY, Kang SY et al (2020) Physiological Importance of Pectin Modifying Genes During Rice Pollen Development. Int J Mol Sci 21 (14). doi:10.3390/ijms21144840

10. Leroux C, Bouton S, Kiefer-Meyer M-C et al (2015) PECTIN METHYLESTERASE48 Is Involved in Arabidopsis Pollen Grain Germination. Plant Physiol 167:367-380. doi:10.1104/pp.114.250928

11. Levesque-Tremblay G, Pelloux J, Braybrook SA, Müller K (2015) Tuning of pectin methylesterification: consequences for cell wall biomechanics and development. Planta 242:791-811.

doi:10.1007/s00425-015-2358-5 
12. Lin S, Dong H, Zhang F et al (2014) BcMF8, a putative arabinogalactan protein-encoding gene, contributes to pollen wall development, aperture formation and pollen tube growth in Brassica campestris. Ann Bot 113:777-788. doi:10.1093/aob/mct315

13. Lin S, Huang L, Yu X et al (2017) Characterization of BcMF23a and BcMF23b, two putative pectin methylesterase genes related to pollen development in Brassica campestris ssp. chinensis. Mol Biol Rep 44:139-148. doi:10.1007/s11033-016-4090-z

14. Livak KJ, Schmittgen TD (2001) Analysis of relative gene expression data using real-time quantitative PCR and the 2-ÄÄCT method. Methods 25:402-408. doi:10.1006/meth.2001.1262

15. Markovič O, Janeček Š (2004) Pectin methylesterases: Sequence-structural features and phylogenetic relationships. Carbohydr Res 339:2281-2295. doi:10.1016/j.carres.2004.06.023

16. Micheli F (2001) Pectin methylesterases: cell wall enzymes with important roles in plant physiology. Trends Plant Sci 6:414-419. doi:10.1016/S1360-1385(01)02045-3

17. Mohnen D (2008) Pectin structure and biosynthesis. Curr Opin Plant Biol 11:266-277. doi:10.1016/j.pbi.2008.03.006

18. Mollet J-C, Leroux C, Dardelle F, Lehner A (2013) Cell Wall Composition, Biosynthesis and Remodeling during Pollen Tube Growth. Plants 2:107-147. doi:10.3390/plants2010107

19. Pelloux J, Rusterucci C, Mellerowicz E (2007) New insights into pectin methylesterase structure and function. Trends Plant Sci 12:267-277. doi: 10.10 16/j.tplants.2007.04.001

20. Roger D, Lacoux J, Lamblin $F$ et al (2001) Isolation of a flax pectin methylesterase promoter and its expression in transgenic tobacco. Plant Sci 160:713-721. doi:10.1016/S0168-9452(00)00456-8

21. Sénéchal F, Graff L, Surcouf $O$ et al (2014) Arabidopsis PECTIN METHYLESTERASE17 is coexpressed with and processed by SBT3.5, a subtilisin-like serine protease. Ann Bot 114:1161-1175. doi:10.1093/aob/mcu035

22. Staunton JR, Fuhrmann A, Nandakumar V et al (2011) PLATFORM AA: Cell and Bacterial Mechanics Motility. Biophysj 100:190a. doi:10.1016/j.bpj.2010.12.1257

23. Tian GW, Chen MH, Zaltsman A, Citovsky V (2006) Pollen-specific pectin methylesterase involved in pollen tube growth. Dev Biol 294:83-91. doi:10.1016/j.ydbio.2006.02.026

24. Tung C, Dwyer KG, Nasrallah ME et al (2005) Genome-Wide Identification of Genes Expressed in Arabidopsis Pistils Specifically along the Path of Pollen Tube Growth 1 [ w ]. Plant Physiol 138:1-13. doi:10.1104/pp.105.060558.cells

25. Turbant $A$, Fournet $F$, Lequart $M$ et al (2016) PME58 plays a role in pectin distribution during seed coat mucilage extrusion through homogalacturonan modification. J Exp Bot 67:2177-2190. doi:10.1093/jxb/erw025

26. Wang H, Zhuang X, Cai $Y$ et al (2013) Apical F-actin-regulated exocytic targeting of NtPPME1 is essential for construction and rigidity of the pollen tube cell wall. Plant $\mathrm{J}$ 76:367-379. doi:10.1111/tpj. 12300 
27. Xiong X, Zhou D, Xu L et al (2019) BcPME37c is involved in pollen intine formation in Brassica campestris. Biochem Bioph Res Co 517:63-68. doi:10.1016/j.bbrc.2019.07.009

28. Yokoyama R, Nishitani K (2001) A comprehensive expression analysis of all members of a gene family encoding cell-wall enzymes allowed us to predict cis-regulatory regions involved in cell-wall construction in specific organs of Arabidopsis. Plant Cell Physiol 42:1025-1033. doi:10.1093/pcp/pce154

29. Yoneda A, Ito T, Higaki T et al (2010) Cobtorin target analysis reveals that pectin functions in the deposition of cellulose microfibrils in parallel with cortical microtubules. Plant J 64:657-667. doi:10.1111/j.1365-313X.2010.04356.x

30. Yu X, Cao J, Ye W, Wang Y (2004) Construction of an antisense CYP86MF gene plasmid vector and production of a male-sterile Chinese cabbage transformant by the pollen-tube method. J Hortic Sci Biotechnol 79:833-839. doi:10.1080/14620316.2004.11511851

31. Yue X, Lin S, Yu Y et al (2018a) The putative pectin methylesterase gene, BcMF23a, is required for microspore development and pollen tube growth in Brassica campestris. Plant Cell Rep 0:0. doi:10.1007/s00299-018-2285-6

32. Yue X, Cao J, Liu T et al (2018b) Characterization and Expression Pattern of a Putative Pectin Methylesterase Gene, BcMF27. 65:364-371. doi: 10.1134/S1021443718030159

33. Zhang D, Shi J, Yang X (2016) Role of Lipid Metabolism in Plant Pollen Exine Development. In: Nakamura Y, Li-Beisson Y (eds) Lipids in Plant and Algae Development. Springer, Cham, pp 315-337

34. Zhang Z, Zhang B, Chen Z et al (2018) A PECTIN METHYLESTERASE gene at the maize Ga1 locus confers male function in unilateral cross-incompatibility. Nat Commun 9:3678. doi:10.1038/s41467018-06139-8

\section{Figures}

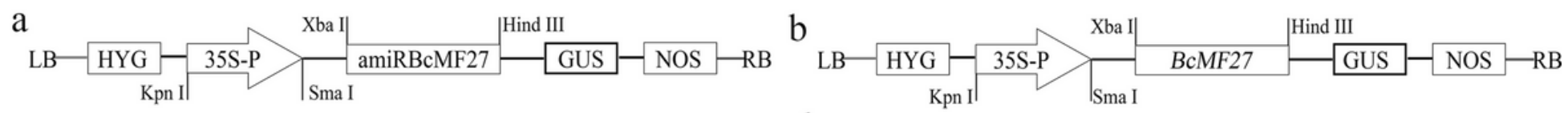

c

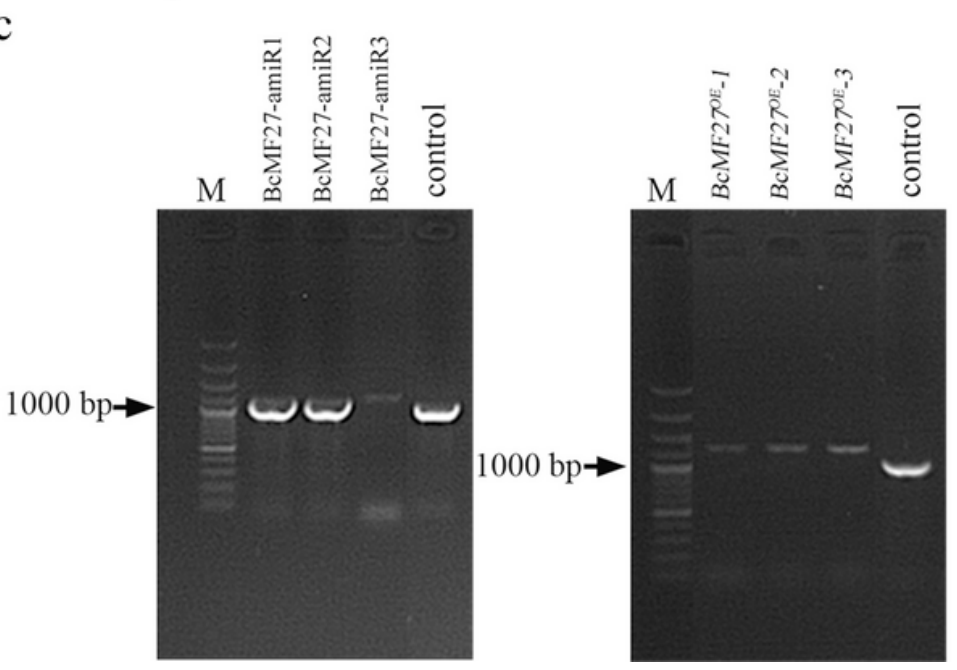

d

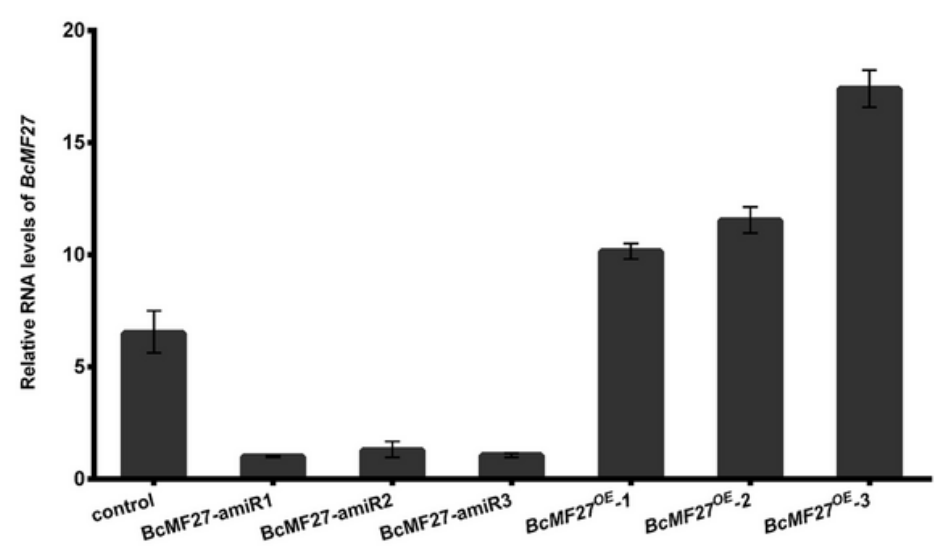


Figure 1

Construction of amiRNA (a) and overexpression (b) vectors of BcMF27 from Brassica campestris ssp. chinensis. (c) PCR detection of positive plants transformed with amiRNA, overexpression and empty vectors. (d) Real-time RT-PCR analysis of BcMF27 in the inflorescences of BcMF27-amiR and BcMF270E transgenic plants. \pm Standard errors for three independent experiments are shown; UBC10 was used as the internal control
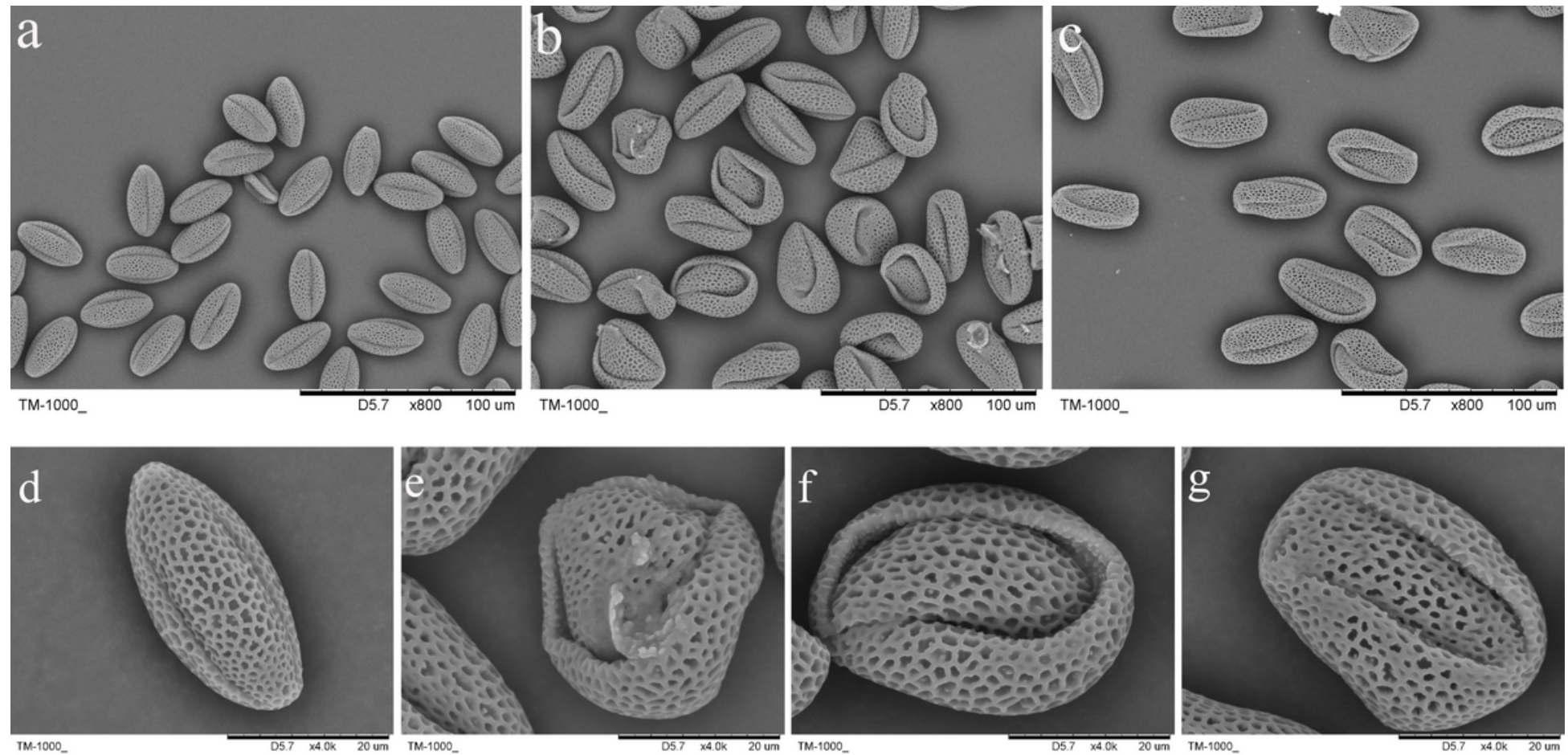

$\mathrm{h}$
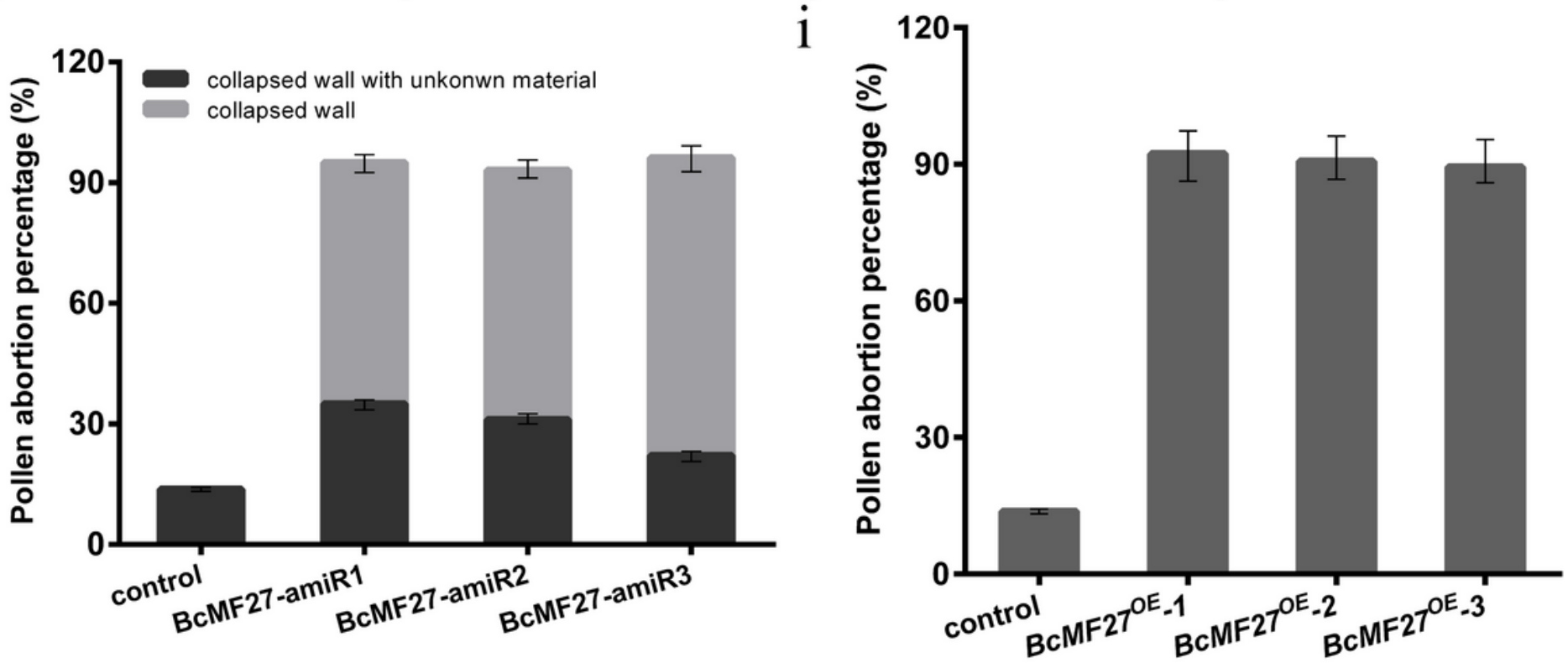

Figure 2

Scanning electron microscopy observation of pollen from BcMF27-amiR and BcMF270E plants. (a-c) Pollen of the control, BcMF27-amiR, and BcMF270E plants, respectively. (d) Magnified images of (a). (e and f) Magnified images of (b). (g) Magnified images of (c). (h and i) Pollen abortion percentage of 
BcMF27-amiR and BcMF270E plants. The values are the mean \pm standard error. Scale bars $100 \mu \mathrm{m}$ in a-c, Scale bars $20 \mu \mathrm{m}$ in $\mathrm{d}-\mathrm{j}$
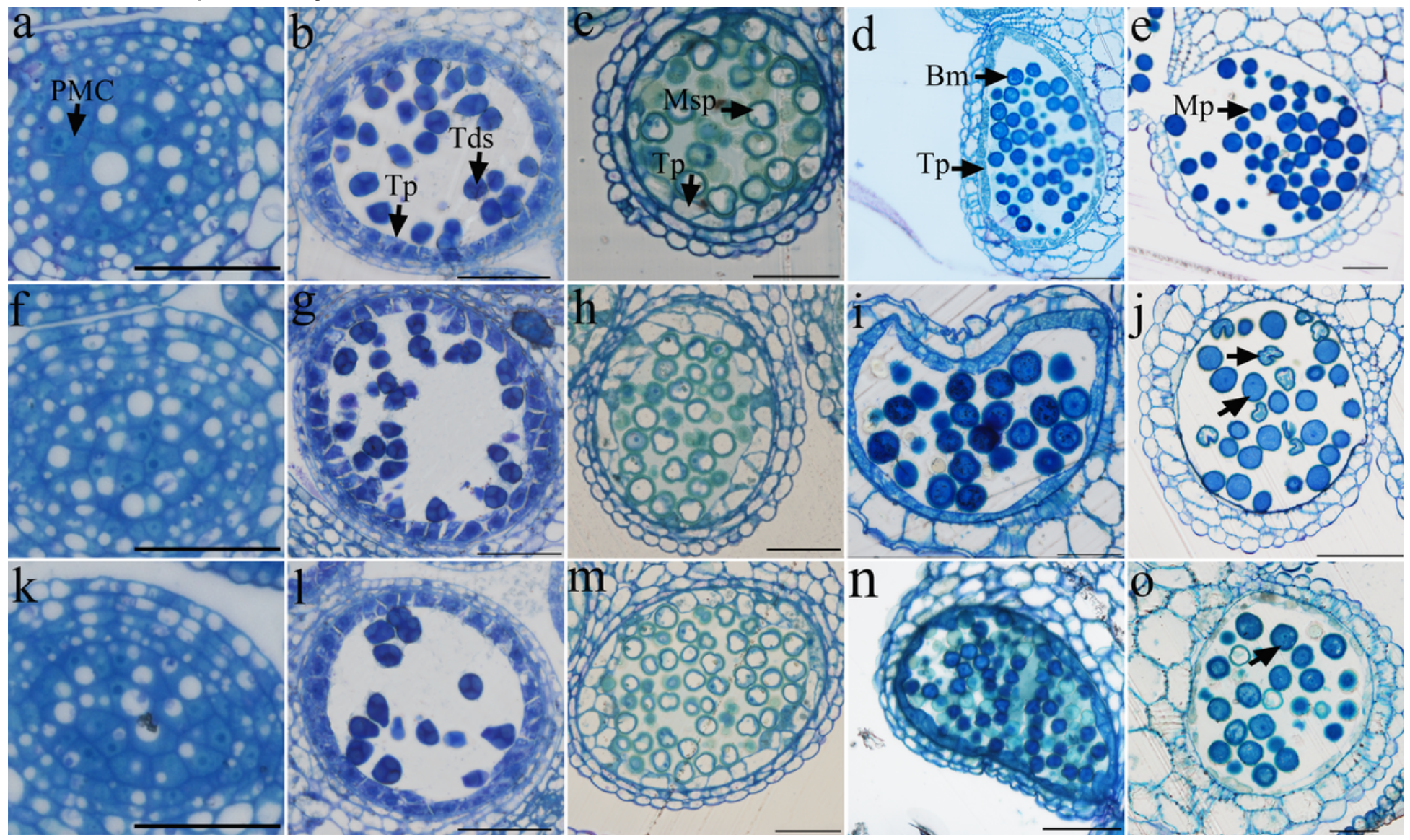

Figure 3

Semi-thin section observation of pollen from BcMF27-amiR and BcMF270E plants. (a-o) Semi-thin sections of anthers in the control $(a-e), B c M F 27-a m i R(f-j)$, and BCMF270E $(k-0)$ plants at the pollen mother cell stage $(a, f, k)$, tetrad stage $(b, g, l)$, uninucleate stage $(c, h, m)$, binucleate stage $(d, i, n)$, and trinucleate stage $(e, j, o)$. PMC, pollen mother cell; Tp, tapetum; Tds, tetreds; Msp, microspore; Bm, binucleate microscope; MP, mature pollen. Scale bars $50 \mu \mathrm{m}$ in a-o 


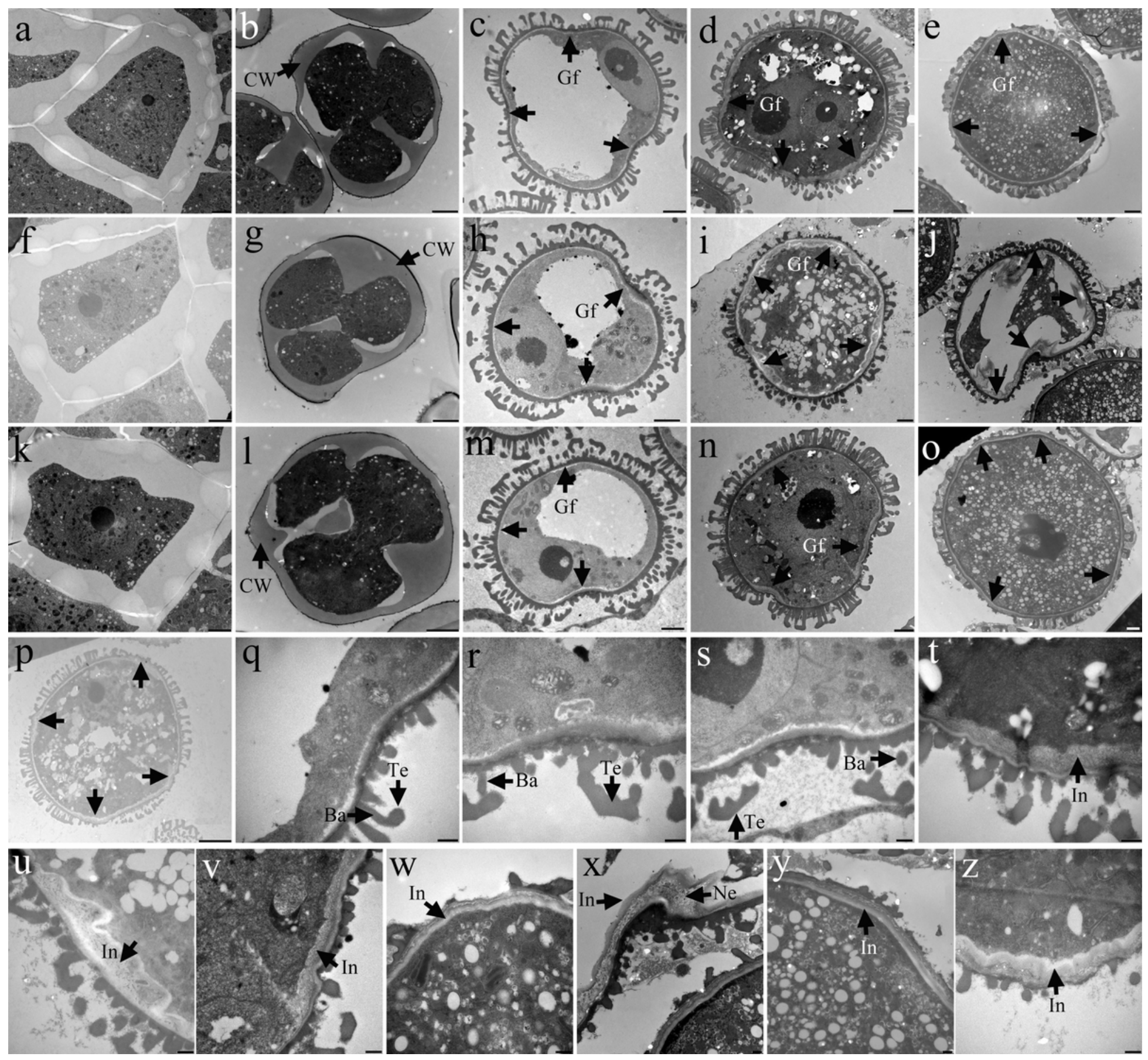

Figure 4

Transmission electron microscopy observation of microspore development in BcMF27-amiR and BcMF270E plants. $(a-p)$ pollen of control ( $a-e)$, BcMF27-amiR ( $f-j$ and p), and BcMF270E (k-o) plants at pollen mother cell stage ( $a, f$, and $k)$, tetrad stage $(b, g$, and $l)$, uninucleate stage (c, h, and $m)$, binucleate stage $(d, i$, and $n)$, and trinucleate stage $(e, j, o$, and p). (q-s) Magnified images of the corresponding aperture region in $c, h$, and $m$. (t-v) Magnified images of pollen wall in $d, i$, and $n$. ( $w-z$ ) Magnified images of the pollen wall in e, j, o, and p. CW, callose wall; Gf, aperture; Te, tectum; Ba, baculum; Ne, nexine; In, intine. Scale bars $5 \mu \mathrm{m}$ in a-p; $2 \mu \mathrm{m}$ in $\mathrm{q}-\mathrm{z}$ 

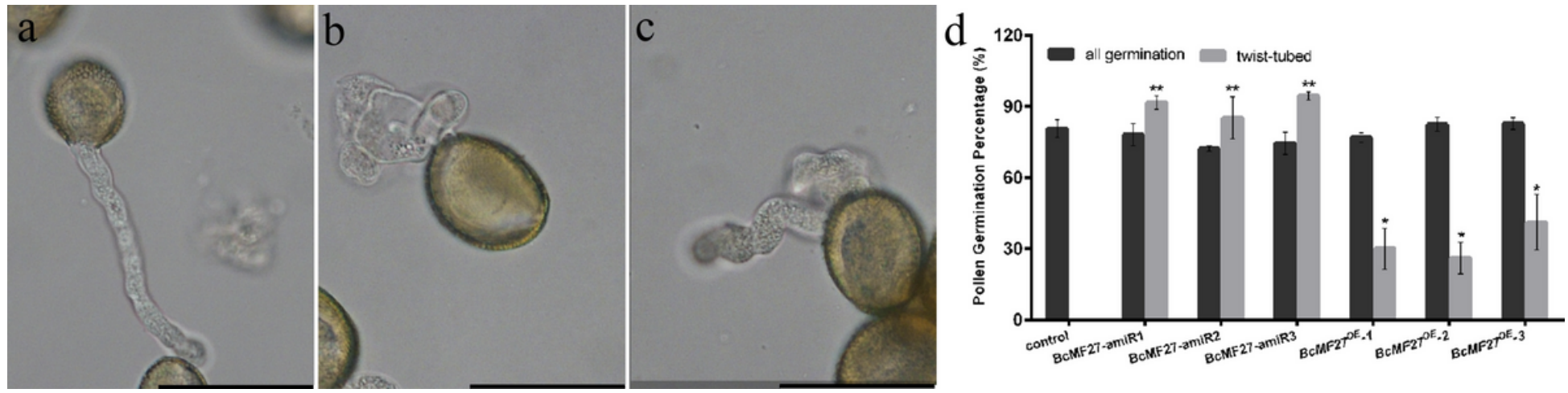

\section{Figure 5}

Pollen germination and pollen tube growth in vitro in BcMF27-amiR and BcMF270E plant. (a-c) Pollen germination of control (a), BcMF27-amiR (b), and BcMF27OE (c) plant in vitro. (d) Pollen germination frequencies of control, BcMF27-amiR, and BcMF270E plants and abnormal pollen tube percentage in all pollen germinated in vitro. ${ }^{*} p<0.05$ and ${ }^{*} \mathrm{p} p<0.01$ vs. the control. The values are the mean \pm standard error. Scale bars $50 \mu \mathrm{m}$

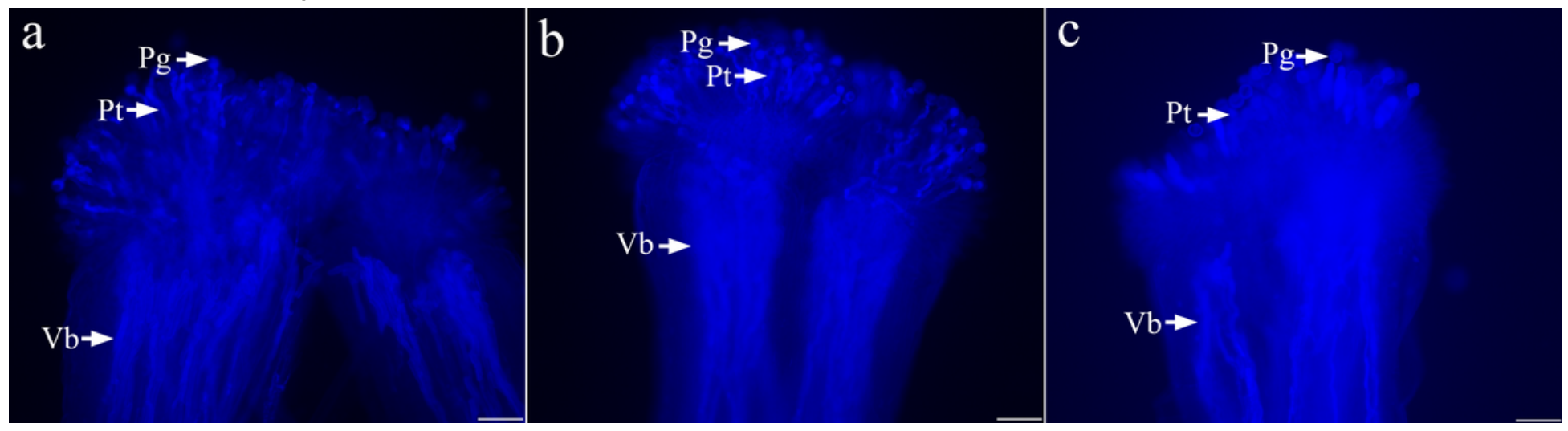

\section{Figure 6}

Pollen germination and pollen tube growth of BcMF27-amiR and BcMF270E on control pistils in vivo. (a) Control pollen tube growth at $4 \mathrm{~h}$ after self-pollination. (b) BcMF27-amiR pollen tube growth at $4 \mathrm{~h}$ after pollination. (c) BcMF270E pollen tube growth at $4 \mathrm{~h}$ after pollination. PG, pollen grains; Vb, vascular; $\mathrm{Pt}$, pollen tube. Scale bars $50 \mu \mathrm{m}$

\section{Supplementary Files}

This is a list of supplementary files associated with this preprint. Click to download.

- Figs1.tif

- FigS2.tiff

- SupplementaryInformation.docx 